\title{
Upshift of the fractional quantum Hall plateaux: evidence for repulsive scattering for composite fermions
}

\author{
Hong Syuan Wang ${ }^{\mathrm{a}}$, Yi Hsing Chiu ${ }^{\mathrm{b}}$, Gil-Ho Kim ${ }^{\mathrm{b}}$, C.-T. Liang ${ }^{\mathrm{a}, *}$, M.Y. Simmons ${ }^{\mathrm{c}, \mathrm{d}}$, \\ D.A. Ritchie ${ }^{\mathrm{c}}$ \\ a Department of Physics, National Taiwan University, Taipei 106, Taiwan \\ ${ }^{\mathrm{b}}$ Department of Electronic and Electrical Engineering, Sungkyunkwan University, Suwon 440-746, South Korea \\ ${ }^{\mathrm{c}}$ Cavendish Laboratory, Madingley Road, Cambridge CB3 OHE, UK \\ ${ }^{\mathrm{d}}$ School of Physics, University of New South Wales, Sydney 2052, Australia
}

\begin{abstract}
It is now established that if there are an equal number of positively and negatively charged impurities, the quantum Hall plateaux in $\rho_{x y}(B)$ are expected to be centred about the classical value $\rho_{x y}=B /(e n)$ where $n$ is the $2 \mathrm{D}$ carrier density. It has also been shown that when repulsive or attractive scattering centres are deliberately introduced close to a two-dimensional electron gas (2DEG), the centres of the quantum Hall plateaux are shifted to higher or lower magnetic field, respectively. In this paper, we present magneto transport measurements on a high-quality GaAs electron system. We extend this concept to the composite fermions where each electron is bound to two magnetic flux quanta. We provide compelling evidence that the dominant scattering for the composite fermions is different from that for electrons. This is supported by studying the upshift of the $v=1$ quantum Hall plateau within the composite fermion picture.
\end{abstract}

(C) 2003 Elsevier B.V. All rights reserved.

PACS: $73.40-\mathrm{c} ; 73.40-\mathrm{f}$

Keywords: Fractional quantum Hall effects; Composite fermion; Repulsive scattering

The fractional quantum Hall effect (FQHE) is observed in high-mobility two-dimensional electron systems in the low-temperature, high-magnetic-field regime [1]. It is believed that the FQHE arises from strong electron-electron interactions, causing the two-dimensional electrons to condense into a fractional quantum Hall liquid [2]. Jain [3] introduced the concept of "composite fermion" where each electron is bound to two magnetic flux quanta, and in this picture the FQHE can then be understood as a

\footnotetext{
* Corresponding author. Tel.: +886-2-23697238; fax: +8862-23639984.

E-mail address: ctliang@phys.ntu.edu.tw (C.-T. Liang).
}

manifestation of the integer quantum Hall effect of composite fermions. At a Landau level filling factor $v=\frac{1}{2}$, a two-dimensional electron system can be transformed into a composite fermion system interacting with a Chern-Simons gauge field $[4,5]$. To date, a wide variety of experiments have demonstrated that at $v=\frac{1}{2}$ the effective magnetic field acting on the composite fermions is zero [6-13]. Away from $v=\frac{1}{2}$, the effective magnetic field acts on the composite fermions is given by $B_{\text {eff }}=B_{\text {ext }}-B\left(v=\frac{1}{2}\right)$ where $B_{\text {ext }}$ is the applied external magnetic field. At $v=\frac{1}{2}$, each electron is bound to two magnetic flux quanta, and thus the density of the composite fermion system is equal to that of the electron system. 


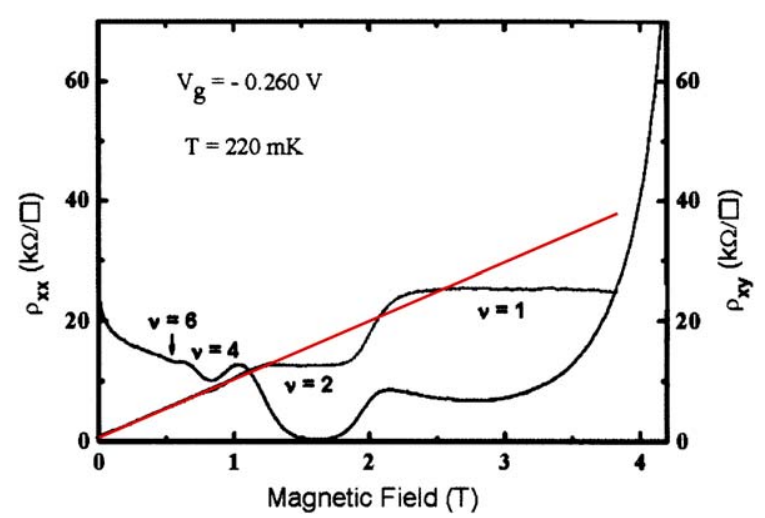

Fig. 1. Four-terminal longitudinal and Hall magnetoresistivity measurements of a two-dimensional GaAs electron system containing self-assembled InAs quantum dots. The solid line represents the extrapolation of the classical Hall effect at low magnetic fields. The centers of the $v=1$ and 2 quantum Hall plateaux are shifted to higher or lower magnetic field.

It is now well established that if there are an equal number of positively and negatively charged impurities, the quantum Hall plateaux in $\rho_{x y}(B)$ are expected to be centred about the classical value $\rho_{x y}=B /($ en $)$ where $n$ is the two-dimensional carrier density. Haug and co-workers [14] have shown that when repulsive or attractive scattering centres are deliberately introduced close to a two-dimensional electron gas (2DEG), the centres of the quantum Hall plateaux are shifted to higher or lower magnetic field, respectively. In order to provide a clear picture of this, in Fig. 1 we show magneto transport measurements on a two-dimensional GaAs electron gas containing self-assembled InAs quantum dots. The solid line represents the linear extrapolation of the low-field classical Hall effect. It is clear that there are upshifts for both $v=2$ and 1 quantum Hall plateaux, showing compelling evidence for strong repulsive scattering in the two-dimensional GaAs electron system due to the introduction of the self-assembled InAs quantum dots.

In this paper, we present magneto transport measurements on a high-quality GaAs electron system. We provide compelling evidence that the dominant scattering for the composite fermions is different from that for electrons. This is supported by studying the upshift of the $v=1$ quantum Hall plateau within the composite fermion picture. In contrast, the centre of the $v=1$ quantum Hall plateau is close to its classical value within the electron picture.

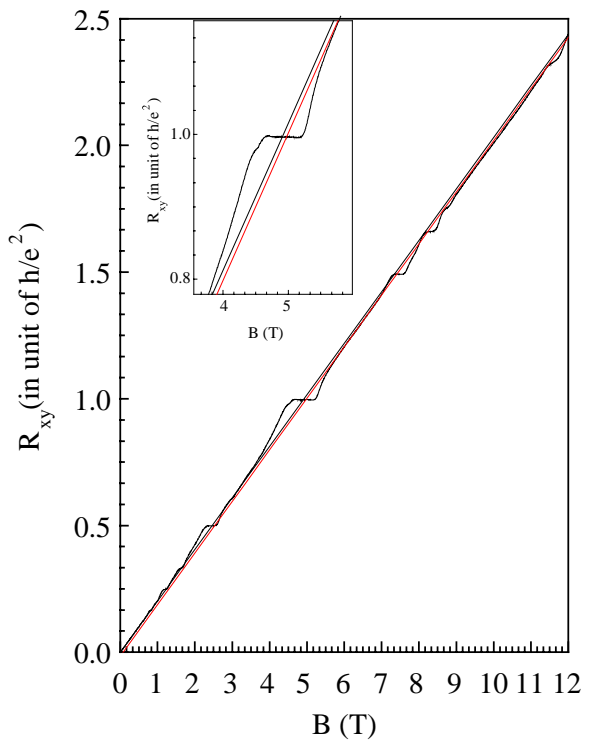

Fig. 2. Hall resistance as a function of perpendicular magnetic field. The linear fit (in the black line) corresponds to the extrapolation of the classical Hall effect for electrons. The linear fit (in the red line) corresponds to the extrapolation of the classical Hall effect for composite fermions. The inset shows the zoom-in of the $v=1$ quantum Hall plateau. For colour figure see the webversion of the article.

The measurements were performed on a Hall bar made from $\mathrm{GaAs} / \mathrm{Al}_{0.33} \mathrm{Ga}_{0.67}$ As heterostructure. The carrier concentration of the $2 \mathrm{DEG}$ was $1.3 \times 10^{11} \mathrm{~cm}^{-2}$ with a mobility of $3.5 \times 10^{6} \mathrm{~cm}^{2} / \mathrm{V} \mathrm{s}$ after brief illumination by a red light emitting diode. The depth of the $2 D E G$ is $300 \mathrm{~nm}$ for our device. Experiments were performed in a top-loading $\mathrm{He}^{3}$ cryostat at $T=300 \mathrm{mK}$ and the four-terminal magnetoresistivity was measured with standard phase-sensitive techniques. A driving current of $10 \mathrm{nA}$ was used in our measurement.

We now turn to our main experimental finding. Within the integer quantum Hall picture, the $v=1$ quantum Hall plateau is centred about the classical value $\rho_{x y}=B /($ en $)$ as clearly shown in Fig. 2. This result clearly demonstrates within the electron picture, there are approximately an equal number of positively and negatively charged impurities within our system. Alternatively, the $v=1$ integer quantum Hall state can be regarded as $v_{\text {eff }}=-1$ integer quantum Hall state for composite fermions where $v_{\text {eff }}$ is the effective filling factor. In this case, the centre of $v_{\text {eff }}=-1$ 
integer quantum Hall state (which is equivalent to the $v=1$ quantum Hall plateau) is shifted to higher effective magnetic field, suggesting that repulsive scattering is stronger than attractive scattering for the composite fermions. The fact that there is a small yet significant difference between the linear fits using two different models provides compelling experimental evidence that repulsive scattering can be more important for composite fermions. For composite fermions, there is an additional scattering due to the random magnetic fields arising from uniform electron distribution [4]. According to the linear fit through $B=0$, the fractional quantum Hall plateaux at $v=\frac{2}{3}$ and $\frac{3}{5}$ appear to shift to the low magnetic field side. This is in contrast to the case for the $v=1$ quantum Hall plateau which is centered around its classical value. However, we would like to point out that it is not valid to consider the up/down shift of a fractional quantum Hall plateau within the electron picture. Alternatively, one should consider the up/down shift of a fractional quantum Hall plateau within the composite fermion picture. In this case, the $v=\frac{2}{3}$ and $\frac{3}{5}$ fractional quantum Hall plateaux are almost centered around the linear fit for composite fermion as shown in the red line.

In conclusion, we have presented magneto transport measurements on a high-quality GaAs electron system. In our system, the Landau level filling factor $v=1$ integer quantum Hall state can also be regarded as $v_{\text {eff }}=-1$ integer quantum Hall state for composite fermions where $v_{\text {eff }}$ is the effective filling factor. We study the centre of the $v=1$ quantum Hall plateau using both the electron picture and the composite fermion picture. We find that within the electron picture, the $v=1$ quantum Hall plateau is centred about the classical value $\rho_{x y}=B /(e n)$. The interesting thing is that within the composite fermion picture, there is a small upshift of the $v=1$ quantum Hall plateau $\left(v_{\mathrm{eff}}=-1\right.$ integer quantum Hall state for composite fermions). These experimental results clearly demonstrate the repulsive scattering is stronger for composite fermions in our system. In the future we would like to measure a high-quality full-gated GaAs electron system. We will measure the upshift of the fractional quantum Hall plateaux at different applied gate voltages to investigate how scattering could be varied at different gate voltage (and hence disorder).

This work was funded by the NSC, Taiwan and the MOE programme for Promoting Academic Excellence of University (89-N-FA01-2-4-3). Gil-Ho Kim was supported by National R\&D Project for Nano Science and Technology (Contract No. M1-0212-04-0003) of MOST. C.T.L. thanks T. Chin for her support.

\section{References}

[1] D.C. Tsui, H.L. Stormer, A.C. Gossard, Phys. Rev. Lett. 48 (1982) 1559.

[2] R.B. Laughlin, Phys. Rev. Lett. 50 (1983) 1395.

[3] J.K. Jain, Phys. Rev. Lett. 63 (1989) 199.

[4] B.I. Halperin, P.A. Lee, N. Read, Phys. Rev. B 47 (1993) 7312.

[5] A. Lopez, E. Fradkin, Phys. Rev. B 44 (1991) 5246.

[6] R.R. Du, H.L. Stormer, D.C. Tsui, L.N. Pfeiffer, K.W. West, Phys. Rev. Lett. 70 (1993) 2944.

[7] R.L. Willett, R.R. Ruel, K.W. West, L.N. Pfeiffer, Phys. Rev. Lett. 71 (1993) 3846.

[8] W. Kang, H.L. Stormer, L.N. Pfeiffer, K.W. Baldwin, K.W. West, Phys. Rev. Lett. 71 (1993) 3850.

[9] D.R. Leadley, R.J. Nicholas, C.T. Foxon, J.J. Harris, Phys. Rev. Lett. 72 (1994) 1906.

[10] L.P. Rokhinson, B. Su, V.J. Goldman, Phys. Rev. B 52 (1995) R11588.

[11] W. Kang, Song He, H.L. Stormer, L.N. Pfeiffer, K.W. Baldwin, K.W. West, Phys. Rev. Lett. 75 (1995) 4106.

[12] J.H. Smet, D. Weiss, R.H. Blick, G. Lütjering, K. von Klitzing, R. Fleischmann, R. Ketzmerick, T. Geisel, G. Weimann, Phys. Rev. Lett. 77 (1996) 2272.

[13] C.-T. Liang, C.G. Smith, D.R. Mace, J.T. Nicholls, J.E.F. Frost, M.Y. Simmons, A.R. Hamilton, D.A. Ritchie, M. Pepper, Phys. Rev. B 53 (1996) 7596.

[14] R.J. Haug, R.R. Gerhardts, K. von Klitzing, K. Ploog, Phys. Rev. Lett. 59 (1987) 1349. 